\title{
An Ordering of Secondary Task Display Attributes
}

\author{
David Tessendorf, C. M. Chewar, Ali Ndiwalana, Jon Pryor, D. Scott McCrickard, Chris North \\ Department of Computer Science \\ Virginia Polytechnic Institute and State University \\ Blacksburg, VA 24061-0106 USA \\ \{dtessend, cchewar, andiwala, jopryor, mccricks, north\}@cs.vt.edu
}

\begin{abstract}
We found that established display design guidelines for focal images cannot be extended to images displayed as a secondary task in a dual-task situation. This paper describes an experiment that determines a new ordering guideline for secondary task image attributes according to human cognitive ability to extract information. The imperative for alternate guidelines is based on the difference in an image's ability to convey meaning, which decreases when moved from a focal to a secondary task situation. Secondary task attribute ordering varies with the level of degradation in the primary task.
\end{abstract}

\section{Keywords}

dual-task, visualization, information design, image attribute

\section{INTRODUCTION}

Computer applications designed to allow user information monitoring and awareness potentially lend enormous efficiency gains within many areas of business, education, and daily life. Displays for multiple or dual-task situations are necessary for applications as simple as instant messengers and news delivery agents, or as vital as vehicular displays, laboratory and security monitors, surgery support and military situational awareness systems. However, ineffectively designed display interfaces, especially dual-task displays, can inhibit, rather than enhance, task performance.

Cleveland and McGill provide an accepted guideline for the presentation of visual data in quantitative tasks, founded on psychophysical theory and experimentation [1]. They recognize visual data as elementary perceptual tasks, described as graph attributes, some of which convey information better than others. Attribute effectiveness guidelines facilitate design of display interfaces that effectively communicate information and create insight. Cleveland and McGill provide an ordering of graph attributes: position along a common scale; position along nonaligned scale; length, direction, angle; area; volume, curvature; shading; and color saturation. Mackinlay extends this list to capture nonquantitative data [2], resulting in the inclusion of more attributes and orderings for nominal and ordinal data.

These experiments establish an ordering of perceptual tasks for print-based media, providing a solid foundation for evaluation of graph design in the focus. It has not been established that the same ordering applies to computer displays or to perceptual tasks required in a dual task situation.

\section{EXPERIMENTAL DESIGN}

In order to empirically test relative attribute effectiveness, a participant plays a simple, yet demanding game on a desktop computer. Scripted, timed events present experimental conditions and record subject performance throughout the experiment. During the game playing, which occurs on the left portion of the screen, a single image with similar dimensions and brightness as the game appears for eight seconds on the right screen edge. The eight-second display time allows data within attended and ignored locations to be reliably and accurately detected [3].

The game playing continues while the subject scans the image for information-the answer to a question asked before the round begins. Each instance of the experiment includes eighteen rounds-nine dual task rounds (gameplaying and image viewing) as well as nine focal (gamefree) rounds. Both treatments require viewing images and answering questions. Participants are 93 undergraduate computer science students, who received class credit.

Six versions of the program implement a Latin square experimental setup testing the independent variables (three attributes, two conditions-single (focal) and dual task). Three base versions differ only in attribute presentation order. Each of these three versions provides two test iterations-one that starts with the dual task and finishes with the focal images, and the other that reverses this sequence.

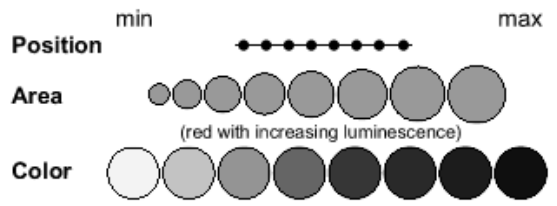

Fig. 1. Attribute scales and encoding schemes used in the experiment are shown. Relative increases within attribute values are uniform. Subjects are shown the applicable scale before the start of each round.

Figure 1 shows attribute scales and encoding schemes. Game rounds cycle through three different question types (identification of displayed minimum/maximum values, 
ratios, or comparison counts) for a single graphically encoded dataset. Regardless of version and attribute encoding, round questions and answers appear in constant order. For instance, images like those in Figure 2 are used in all six versions as the first graph type, but the question ("what is the minimum value?") and answer (A) do not vary. However, two versions (one version displaying the graph as a focal task, the other as a secondary task) encode this first dataset with position, two others use color, and the final two use area. After testing a single graph with three questions, the dataset and encoding scheme change.
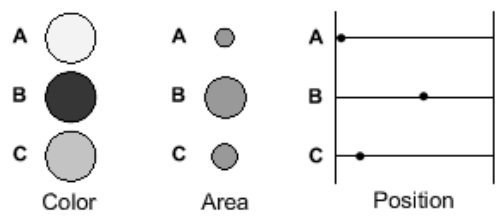

Fig. 2. Each of the three attribute images (Color, Area, Position) encodes the same dataset values $\{1,5,2\}$. In a given round, one of these images is presented as a secondary task while the participant plays a game. Only three values are shown here, but the experiment's images encode ten.

\section{RESULTS}

This study empirically extends focal attribute ordering to computer displays - position is best, then area, and finally color $(\mathrm{F}(2,277)=7.91, \mathrm{MSE}=0.41, \mathrm{p}=0.0005)$. However, secondary tasks show differences-user ability to gain insight from an image is better when displayed in the focus rather than as a secondary task (answer correctness $z$-scores range from 13.189 to $1.965, n=93$ ).

To logically evaluate a secondary task in a dual-task situation, performance effect on both tasks must be considered. Primary task degradation expresses change in game performance during image display period in relation to average game performance before and after the image appears. Answer correctness (secondary task measure) is evaluated for any round meeting a given primary task degradation threshold (acceptable degradation).

Using this dual-task scoring method, data relating answer correctness to attribute types across degradation levels is apparent (Figure 3). Higher percentages of correct answers always result from position-encoded images. However, at low degradation levels, color-encoded images convey insight more often than area-encoding. The opposite condition is true at higher levels of degradation.

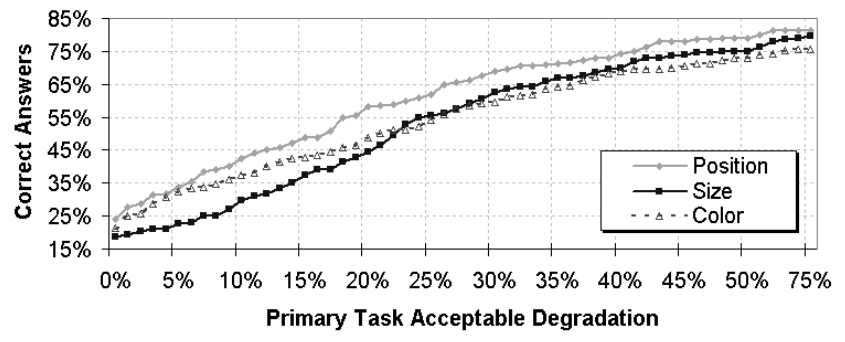

Fig. 3. Percentage of correct answers for each attribute type, at increasing levels of primary task acceptable degradation.

Regressed trendlines (sixth order) show significance of between-group difference in communication of secondary task information encoded with these three attributes (Figure 4). Ordering of attribute effectiveness varies with acceptable degradation and can be completely ordered at low degradation levels-position, color, and then area. Confidence levels for this ordering are established with multivariable analysis of variance (ANOVA) tests.

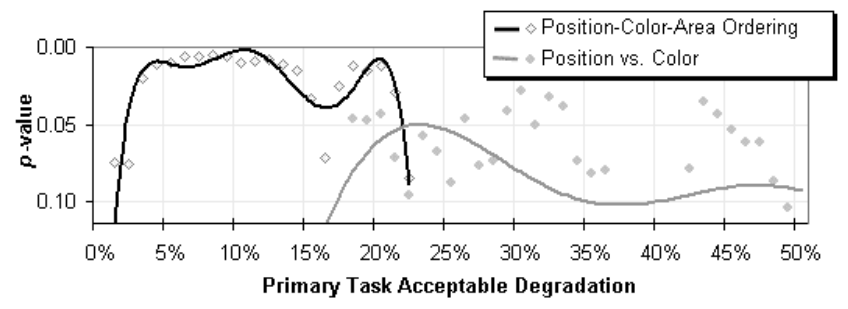

Fig. 4. Solid trendlines show that levels of significance for attribute ordering vary with degree of acceptable primary task degradation. In rounds with minimal primary task degradation (no more than 23\%), subjects answered more questions correctly with position than color images, although both allowed more correct answers than size images.

Several conclusions are evident from these results. First, considering the superiority of position in all focal and dual task orderings, information should be conveyed in terms of relative position whenever possible to allow optimal probability for accurate communication and primary task sustainability. Secondly, design guidelines-other than those for focal conditions - must address diminished image attribute effectiveness in secondary tasks. Thirdly, it is critical that secondary task display attributes are selected based on specification of acceptable amounts of primary task performance degradation. Designers of vehicular displays and other systems supporting a critical primary task would certainly want to consider attribute ordering at the lowest levels of acceptable primary task degradation. Therefore, secondary task display design should be guided by relevant attribute ordering in Figure 4.

Further work should include evaluation of other display attributes and combinations of attributes in dual-task situation. Since this experiment limited color encoding to incremental instances of red luminescence, it would also be useful to investigate relative effectiveness of other hues. Other attributes examined here may also have better encoding schemes. Guidelines for all facets of interface design should be tested and established for secondary tasks.

\section{REFERENCES}

1. Cleveland, W. S. \& McGill, R. Graphical perception: Theory, experimentation, and application to the development of graphical methods. Journal of American Statistical Association 79, 387 (Sept 1984), 531- 554.

2. Mackinlay, J. Automating the design of graphical presentations of relational information. ACM Transactions on Graphics 5, 2 (April 1986), 110-141.

3. Weber, T., Kramer, A. \& Karni, O. Tracking visual attention with event-related brain potentials, in $A R L$ Advanced \& Interactive Displays Symposium (February 2-6, 1998). 33-38. 